\title{
Castellano amazónico peruano e identidad en Facebook. \\ Análisis lingüístico de la página "En la selva dicen"
}

\section{Peruvian Amazonian Spanish and Facebook identity. \\ Linguistic analysis of the page "En la selva dicen" (In the jungle they say)}

Stefano Pau ${ }^{1}$

Università degli studi di Cagliari (Cerdena, Italia)

\section{Resumen}

Se propone un análisis lingüístico y sociolingüístico de la página de Facebook "En la selva dicen", una comunidad en línea que recopila y publica frases en las que se emplean elementos típicos de la variante amazónica del castellano peruano. El objetivo del estudio es examinar las peculiaridades fonológicas, morfosintácticas y lexicales del castellano amazónico peruano, las maneras como esta variedad es percibida y modificada y, sobre todo, el rol de las redes sociales en la

1 Profesor contratado, Università degli studi di Cagliari (Cerdena, Italia). Contacto: pau.stefano@ymail.com 
preservación y creación de una identidad compartida, alejada de estereotipos y prejuicios.

Palabras clave: En la selva dicen, castellano amazónico peruano, sociolingüística, identidad, análisis morfosintáctico, análisis fonético

\section{Abstract}

This paper proposes a linguistic and sociolinguistic analysis of the Facebook page "En la selva dicen", an online community that collects and publishes phrases using typical elements of the Amazonian variety of Peruvian Castilian. The purpose of the study is to examine the phonological, morphosyntactic and lexical features of the Peruvian Amazonian Castilian, the ways in which this variety is perceived and changed and, above all, the role of social networks in its preservation and creation of a shared identity, without stereotypes and prejudices.

Key words: En la selva dicen, Peruvian Amazonian Castilian, sociolinguistics, identity, morphosyntactic analysis, phonetic analysis...

\section{Introducción}

En este artículo se propone un análisis lingüístico y sociolingüístico de la página de Facebook "En la selva dicen” (www. facebook.com/enlaselvadicen/), una comunidad en línea seguida por 184.515 personas, que recopila y publica frases 
enviadas por los usuarios en las que se emplean elementos típicos de la variante amazónica del castellano peruano.

Los administradores de la página reivindican su orgullo por ser amazónicos, abogan por el fortalecimiento de una identidad amazónica compartida a través del uso de la lengua y rechazan los estereotipos y los estigmas.

Gustavo Solís Fonseca (2009, p. 312), citando un estudio realizado por el Instituto del Bien Común (2006), indica que la región amazónica peruana constituye el $61 \%$ del territorio estatal y comprende los departamentos de Amazonas, Loreto, Madre de Dios y Ucayali, además de porciones más o menos extensas de los departamentos de Cajamarca, San Martín, Huánuco, Cuzco, Puno, Pasco, Junín y Apurímac.

Debido a la inmensidad de este espacio, a nivel lingüístico el panorama resulta particularmente variado, tanto que no existe concordancia en las opiniones de los especialistas siquiera sobre el número de familias lingüísticas existentes en la región. Lo que sí parece fuera de cualquier duda es la progresiva desaparición de varios idiomas, que día a día van perdiendo terreno frente al pujante avance del castellano ${ }^{2}$. Según una estimación plausible de Solís Fonseca (2009, p. 317), actualmente existirían en la Amazonía un total de 40 lenguas, 39 de las cuales formarían parte de 17 familias lin-

2 Aun subrayando lo mucho que queda por hacer, hay que remarcar los esfuerzos que en los últimos años se están llevando a cabo para la revitalización de los idiomas autóctonos con la normalización de los alfabetos, la redacción de gramáticas y diccionarios y los varios proyectos de fortalecimiento de la identidad lingüística. 
güísticas indígenas ${ }^{3}$ y una, es decir el castellano, pertenece a la familia indo-europea.

El objetivo de este estudio es examinar las peculiaridades del castellano hablado en la región amazónica peruana en las dimensiones fonológicas, morfosintácticas y lexicales, las maneras en las que esta variedad es percibida y modificada y, sobre todo, el rol de las redes sociales en su preservación.

\section{El castellano amazónico peruano (CAP)}

Casi cinco siglos de contactos entre las poblaciones indígenas y la población de ascendencia europea han provocado, obviamente, una alteración del horizonte idiomático, tanto de las lenguas autóctonas como de la lengua importada del Viejo Continente. Sin embargo, a lo largo de mucho tiempo, los dos universos -no solo en el área amazónica, sino en todo el país- se habían considerado separados e impermeables a las influencias recíprocas:

[...] se instituyó, con respecto a la realidad lingüística del país, una imagen que calza con la por mucho tiempo supuesta estructura dual de la sociedad que emergió del contacto iniciado por los hombres de Pizarro. Y, combinado lo anterior con un sentimiento de identidad, común entre los criollos, mestizos y sectores urbanos, paulatinamente se afirmó una actitud ante la lengua. Solía ésta contemplar el castellano o español del Perú, nuestro castellano, como una entidad incontaminada y aparte del resto del cuerpo cultural no-hispánico del país. (Escobar, 1978, p. 21)

3 Arawa, arawak, bora, cahuapana, candoshi, harakmbut, jívaro, munichi, pano, peba yagua, quechua, shimaco, takana, tikuna, tukano, tupí, witoto. 
Para cualquier hispanohablante que pise por primera vez los territorios de la selva amazónica peruana, sus pueblos y ciudades, sería imposible afirmar que el castellano hablado en esa región sea efectivamente incontaminado o que tenga las mismas peculiaridades de la lengua llegada con las expediciones y entradas ${ }^{4}$ del siglo XVI y XVII.

Volviendo a las palabras de Alberto Escobar, parece útil citar su punto de partida a la hora de analizar las características reales del castellano hablado en el Perú y, en este caso, en las regiones amazónicas del país:

Postulamos que el estudio de nuestro castellano o español tiene que hacerse aceptándolo como el fruto de un proceso secular, en el cual su perfil se caracteriza no sólo por derivar de su origen europeo, sino por su relación permanente en la dinámica que ha ido forjando lo que es el Perú de hoy. Entre estos factores, no desdeñaremos la situación multicultural y multilingüe del Perú. (1978, p. 22).

Joaquín García identifica esos factores entremezclando elementos sociolingüísticos con palabras evocadoras que, sin embargo, pueden resultar provechosas a la hora de analizar la vivacidad léxica y los elementos suprasegmentales, como la entonación de la peculiar forma de hablar amazónica; para él, el castellano amazónico (y en particular loretano):

[...] reasume su pasado; recoge a modo y medida el viejo decir de Castilla; integra miles de vocablos y componentes ideológicos del kichwa, que en su tiempo fue la lengua franca y vehículo de comunicación interétnico; y lo adorna todo con el sabroso néctar que sube desde los ríos, la inmensi-

4 Así se llamaban las expediciones con las que se emprendía la penetración colonial en la selva (Varese, 1973, pp. 101-103). 
dad de la flora, y todos los seres vivientes. Así el selvático ha llegado a componer una suerte de dialecto tan vivo y chispeante como es el mar de musicalidad que lo envuelve. (García, 2015, p. 5)

Cabe mencionar que la definición de dialecto empleada por García no conlleva ningún matiz negativo o despectivo, pues está claro que a nivel lingüístico no existe ninguna forma para diferenciar una lengua de un dialecto (Berruto, 2004: Matthews, 1979), a no ser que se tomen en cuenta los aspectos ligados al prestigio y las dimensiones (Hudson, 1998, pp. 38-43). Las diferencias son, por ende, sociolingüísticas, puesto que "Cuando hacemos referencia a las variedades del español según la región geográfica, decimos que estamos hablando de los dialectos de la lengua espańola. El dialecto es, entonces, una variedad lingüística definida por las características regionales de sus hablantes. Es decir, es una variedad lingüística regional" (Escobar, 2010, pp. 392-393).

Alberto Escobar (1978, p. 38) prefiere utilizar el término variedad para evitar cualquier equivocación, "subrayar su connotación dominantemente sociolingüística y eludir los malentendidos que circulan con la voz dialecto”. Explica, además, su división en zonas de las variedades del castellano peruano y explica que la zonificación intenta demarcar las áreas espaciales en las que prevalece una determinada variedad (a pesar de la posible existencia, en la misma área, de otras distintas).

En efecto, hablar de castellano amazónico o del español peruano amazónico 5 podría desviarse de un análisis preci-

5 Algunos estudios realizados sobre todo en lo Estados Unidos (García, 2011; Jara Yupanqui, 2012; Jara y Valenzuela, 2013; Vallejos, 2014) emplean la definición de Peruvian Amazonian Spanish (PAS). En este 
so, puesto que el área geográfica que comúnmente se llama Amazonía resulta ser inmensa y comprende lugares alejados el uno del otro en los que las formas de hablar pueden variar de forma contundente. Por esta razón, algunas de las principales investigaciones de lingüística amazónica prefieren restringir el objeto de estudio a las mayores áreas urbanas de la región, es decir, a los alrededores de las ciudades de Iquitos (Barraza, 1998; Pérez Falcón, 2015) y Pucallpa, capitales de los departamentos de Loreto y Ucayali, respectivamente, o a situaciones peculiares, como los casos de migración de habitantes indígenas amazónicos en contextos ciudadanos (Falcón Ccenta 2012; Falcón, Chumbile y Canturín, 2012). Así, Margarita Jara Yupanqui se centra en el español loretano (Loretan Spanish, LS), que define como la variedad usada en el departamento de Loreto (y en particular en Iquitos, centro de influencia de toda la región), comprendida en el interior del gran contenedor del CAP, término empleado como un paraguas para referirse tanto a las variedades habladas en las tierras bajas como en las tierras altas y la ceja de selva (2012, p. 448).

Al contrario, trabajos de distinta índole, como el reciente Diccionario amazónico compilado por el antropólogo Alberto Chirif, intentan ensanchar la mirada hacia un panorama unificador, puesto que:

Loreto como espacio administrativo particular no ha tenido siempre la misma configuración actual sino que ha sido una realidad cambiante. [...] Estas desmembraciones territoriales ${ }^{6}$ hacen ver cuán arbitrario habría sido limitar el alcance

artículo, se utilizará la denominación castellano amazónico peruano o su acrónimo CAP.

6 Se refiere a la división del antiguo departamento de Loreto en Loreto y San Martín (1906) y a la que separó el actual departamento de Ucayali en 1981. 
de este diccionario a un espacio físico que se ha ido conformando por circunstancias administrativas que no han significado una barrera para las dinámicas sociales y culturales que son anteriores a él y que se han mantenido después de la secesión. (2016, p. 14)

Efectivamente, a pesar de las obvias diferencias debido a las distancias geográficas es posible rastrear algunas características lingüísticas comunes a lo largo de toda la región geográfica amazónica. Cabe preguntarse a qué razones se debe tal uniformidad; una posible respuesta podría ser abordar la cuestión desde un punto de vista diacrónico y analizar-como lo hacen Luis Hernán Ramírez (2003), José María Arroyo (1977), Thatiana Pérez Falcón (2015) o Manuel Marticorena (2010), entre otros- cómo y cuándo ha aparecido el español en la selva.

\section{"El castellano aparece en la selva como una explosión”}

Las primeras huellas de la lengua española en el espacio oriental del actual Estado peruano datan de los primeros ańos de la Conquista, puesto que las primeras entradas empezaron a partir de 1530, cuando se emprendió la exploración de la vertiente oriental de los Andes y de la región de la ceja de selva siguiendo el curso de los ríos que bajaban de las montańas.

260 A partir de las primeras décadas de la presencia hispánica se fueron fundando algunas de las principales ciudades de la floresta ${ }^{7}$, pero el avance de las tropas virreinales en la mayoría de los casos no fue fácil y muchas de las expediciones terminaron en fracasos totales. La infructuosidad de las maniobras militares, obviamente, se reflejó también a nivel lingüístico.

7 Entre ellas, Moyobamba (h 1540) y Jaén (1949) (Ramírez, 2003). 
La situación no cambió sustancialmente con el comienzo de la penetración religiosa y la implantación del sistema de las reducciones $^{8}$. Las órdenes religiosas que más se empeñaron en la obra misionera fueron los franciscanos y los jesuitas. Su trabajo, en realidad, fue muy complejo por lo menos hasta los últimos ańos del siglo XVII, cuando retomaron vigor e intensificaron la fundación de caseríos en los que se reunían personas de distintas etnias ${ }^{9}$, por lo que frecuentemente la comunicación presentaba muchas dificultades. Para hacer frente a este problema, a nivel educativo, los misioneros jesuitas enfatizaron la enseñanza de la lengua 'inga', el quechua, para facilitar a los indígenas la comprensión recíproca y, factor no menos importante, para ahorrarse la tarea de aprender las numerosas lenguas de sus 'protegidos'. De esta forma, empezó una primera nivelación de las culturas amazónicas y la progresiva desaparición de varias de ellas. Rosa Vallejos (2014, p. 427) señala que en los últimos años antes de la expulsión de los jesuitas (1767) en algunas zonas también se

8 Aunque el objetivo primario apuntaba a la extirpación de las supersticiones y a la catequización de los moradores amazónicos, en muchos casos, las expediciones misioneras también surgían de la esperanza de encontrar las supuestas riquezas escondidas en el interior de la selva, de las que el legendario El Dorado buscado por Pedro de Ursúa y Lope de Aguirre representa el ejemplo más notorio, loco y, a pesar de esto, más paradójicamente anclado en el imaginario de varias generaciones de aventureros.

9 El historiador Jesús de San Román, citando al padre Manuel Uriarte, pone el ejemplo del poblado de San Joaquín de Omaguas, que se encontraba originariamente en los alrededores de la confluencia entre el río Ampiyacu y el río de las Amazonas y luego se transfirió dos veces hasta la actual colocación entre las ciudades de Iquitos y Nauta, que albergaba, según las palabras del misionero jesuita, alrededor de seiscientos personas entre omaguas, yurimaguas, migueanos, amaonos, mayorunas, cocamas y cocamillas (San Román, 1975, p. 52). 
emplearon con la misma finalidad el omagua y el kukama, de la familia tupí.

Manuel Marticorena apoya la tesis de Teddy Bendayán acerca del por qué los jesuitas no utilizaron el castellano para llevar a cabo su labor evangelizadora; la razón tendría que ver con la variada procedencia de los religiosos, puesto que "en la orden habían [sic] españoles, holandeses, alemanes y de otras nacionalidades" (Marticorena, 2010, p. 42) ${ }^{10}$.

Sea cual fuere la razón, parece fuera de cualquier duda la escasa presencia del espańol en el panorama amazónico por lo menos hasta la segunda mitad del siglo XIX, cuando se aprobaron algunas medidas que impulsaban la colonización de la selva (Escobar, 1978, p. 73; Santos Granero y Barclay, 2002, p. 30), tanto por parte de inmigrantes europeos, como de los habitantes de las regiones andinas, lo que favoreció la movilidad al interior de país.

La llegada de nuevos colonos hispanohablantes alcanzó números impresionantes a partir del último cuarto del siglo, debido al auge del caucho. En esa temporada, "El castellano aparece en la selva como una explosión, bruscamente, sin prehistoria. [...] inútil buscar la sucesión gradual. No la hay. [...] El fenómeno del castellano es una 'epifanía' sin 'navidad'. Es una erupción súbita de un volcán lingüístico que entra en actividad" (Arroyo, 1977).

10 Pérez Falcón Ccenta (2015, pp. 29-30) señala que en las crónicas de los franciscanos no se hace ninguna referencia al empleo del quechua como lengua franca y sí se menciona el uso del castellano, subrayando, sin embargo, los escasos resultados de esta imposición. 
La necesidad de comunicar, el nacimiento de nuevas ciudades y el crecimiento exponencial de otras, y el uso de la violencia en las relaciones laborales con los indígenas (Marticorena, 2010, p. 43) llevó a una rápida difusión del idioma llegado siglos antes de Europa. Los hispanohablantes se fueron multiplicando, sobre todo en el ambiente urbano y en las comunidades ribereñas, y llegó incluso a algunos rincones alejados del interior de la selva.

A lo largo de todo el siglo XX otros factores influenciaron la difusión (y también las transformaciones) del castellano en la selva; entre ellas, cabe mencionar por lo menos la mayor rapidez en las conexiones espaciales con la creación de la carretera Lima-Pucallpa y el establecimiento de las rutas aéreas, la llegada de inmigrantes debido al comienzo de la explotación petrolera y, en los últimos años, la difusión de medios de comunicación masiva como la televisión o el internet.

Para Alberto Escobar (1978, p. 75), algunas de las peculiaridades del castellano amazónico se deberían justamente a su aislamiento del resto del país, a su condición periférica respecto de los centros políticos y administrativos, al influjo a nivel de substrato de las lenguas indígenas y a sus lazos con las regiones colindantes occidentales, es decir, a los contactos con los lusohablantes de Brasil.

\section{Características del castellano amazónico}

El mismo Escobar saca estas conclusiones después de un cuidadoso estudio sobre las características fundamentales de las formas de hablar de los peruanos y las peruanas. En su trabajo de 1978 titulado Variaciones sociolingüísticas del cas- 
tellano en el Perú, consciente de la necesidad de abordar el fenómeno lingüístico desde una perspectiva que incluya el contexto socioeconómico (1978, p. 19), el filólogo plantea una primera división entre los y las hablantes de español como L2 -es decir, los y las hablantes bilingües que tienen como L1 una lengua indígena-, y los que lo tienen como lengua materna.

Su análisis se centra en estos últimos y toma como punto de partida la diferencia en la pronunciación del fonema lateral palatal $/ K /$-que en un sistema conservador (lleísta) correspondería a la grafía -1l-- y de la fricativa dorsopalatal /j/, que correspondería a la grafía -y-, que en muchas variantes dialectales, llamadas yeístas, ha sustituido a la lateral.

A partir de esta diferencia, Escobar (1978, p. 40) divide el país en dos tipos lingüísticos, compuestos por un total de cinco variantes o dialectos: el castellano de tipo 1, que el autor identifica como castellano andino, es el que mantiene la diferencia entre los dos sonidos y estaría conformado por la variante andina propiamente dicha, por la altiplánica y por la variedad del litoral y de los Andes suroccidentales. El castellano de tipo 2 o castellano ribereño o no andino, por su parte, sería un tipo yeísta y estaría integrado, de un lado, por la variedad del litoral centro-norteńo y central y, del otro, por

264 el castellano amazónico.

Entre las características fonéticas peculiares de la variedad amazónica, Escobar subraya principalmente la tendencia hacia la africación y el adelantamiento de /j/, que pasa a pronunciarse como una africada postpalatal sonora /dz/; la frecuente desafricación de $/ \mathrm{t} f /$ que pasa a pronunciarse como una fricativa postpalatal $/ \delta / \mathrm{y}$, por último, la neutralización 
de la fricativa velar / $\mathrm{x} / \mathrm{y}$ labiodental /f/ en el archifonema fricativo bilabial sordo $/ \phi /$.

Luis Hernán Ramírez (2003), por su parte, propone una división dialectal que concuerda más con la tradicional tripartición geográfica del espacio peruano en costa, sierra y selva, e identifica una variante andina, una costeńa o del litoral y una amazónica. El autor, a diferencia de Escobar, sostiene -basándose también en datos procedentes de análisis fonéticos- que la variante amazónica tiene más coincidencia con el habla andina que con la del litoral. Entre otros factores, habría concordancia, por ejemplo, en la conservación del fonema /s/ antes de consonante (que en la variedad costeńa tendería hacia la velarización o incluso hacia la aspiración) y en el sistema vocálico, influenciado de manera contundente por el substrato quechua.

Otro escrupuloso trabajo que aborda desde varios aspectos el castellano amazónico es el de Manuel Marticorena publicado en 2010 y titulado El castellano Amazónico del Perú. El autor, aun subrayando su amplia difusión tanto en la zona de selva baja (principalmente en el actual departamento de Loreto) como en la selva alta (Ucayali, San Martín y Madre de Dios), destaca la importancia de la ciudad de Iquitos en la difusión de la variedad lingüística en análisis. La capital loretana, de hecho, actuaría -tanto hoy como en el pasado- a la vez como centro de confluencia de las distintas variantes subregionales y como foco irradiador, por ser el núcleo donde tiene lugar el mayor desarrollo y desde donde se propagan las innovaciones.

En el plano fonético, Marticorena concuerda con Ramírez al afirmar que muchas de las particularidades vocálicas y 
consonánticas son asimilaciones del quechua amazónico; sin embargo, el aspecto más interesante del estudio de Marticorena reside en su acercamiento pluridimensional, pues no se limita al aspecto fonético, sino que explora también desde la óptica morfológica, sintáctica, lexical, semántica y de la entonación.

A la vez, el autor puntualiza que:

[...] este castellano amazónico en los medios urbanos como Iquitos y Pucallpa está en un proceso de desaparición, evolucionando a nuevas formas de castellano, mientras en los poblados donde la presencia es rural netamente, se mantiene su estructura [...], siendo mínimo su proceso de variación por el hecho de ser menor la influencia externa. (2010, p. 48)

La percepción de una progresiva pérdida de las peculiaridades lingüísticas amazónicas es confirmada también por trabajos llevados a cabo en años recientes por dos investigadoras peruanas radicadas en Estados Unidos. Margarita Jara Yupanqui (2012, pp. 453-454), en primer término, subraya el hecho de que la consciencia que los loretanos y las loretanas (o mejor dicho, los iquiteños y las iquiteñas) tienen de su peculiar forma de hablar se debe principalmente a la visión que sobre ellos llega del exterior (en particular de Lima) y tiene que ver principalmente con la entonación: "Nos dicen que tenemos la forma de hablar cantando [...]; a diferencia de los otros dialectos de la costa, aquí sí tienen una entonación muy diferente. [...] Nosotros hablamos más lento que los de la costa por eso nos dicen 'charapas'11" (Jara Yupanqui,

11 Alberto Chirif (2016, p. 97) propone dos entradas para el término 'charapa'; en la primera, explica que se trata de un animal (Podocnemis 
2012, pp. 454-455) son algunas de las declaraciones de los entrevistados por la autora.

Sin embargo, se subraya que los entrevistados más ancianos perciben en la forma de hablar de los jóvenes una diferencia generacional, que los lleva a perder algunas de las peculiaridades y a uniformarse hacia una variante que es percibida como más correcta u oficial: el habla limeña. Este cambio, según la investigadora, sería facilitado por la frecuente visión estereotipada y estigmatizada del habla amazónica y conllevaría, por ejemplo, a la pérdida de elementos lexicales procedentes de los idiomas autóctonos (tanto del quechua como de las lenguas amazónicas), a su arrinconamiento en el ámbito doméstico o a la percepción de ellos, por parte de los jóvenes, como 'graciosos' ${ }^{12}$. Las razones de esta mutación en las jóvenes generaciones iquiteñas podrían radicar, según los colaboradores de la autora, en un proceso de aculturación causado por la globalización o por la idea de que cambiar la forma de hablar permite mejorar el estatus social. También se menciona el rol de los medios de comunicación, que han ensanchado la brecha entre las generaciones. Estos factores llevarían, por ende, a una nivelación lingüística que no solo podría causar la definitiva desaparición de los idiomas indígenas más vulnerables, sino también a una pérdida de las características del castellano que ha evolucionado de forma tan peculiar en el espacio selvático.

expansa) y "es la más grande de las tortugas fluviales de toda la América", mientras que en la segunda dice que hoy en día ha sufrido una resemantización y es un "gentilicio popular para designar a la gente de Loreto y también de Ucayali".

12 No solo graciosos, sino también antiguos, indígenas (en sentido despectivo), informales o incultos (Jara Yupanqui, 2012, p. 459). 
Rosa Vallejos (2014) también subraya que en algunos casos -como para la permutación de los fonemas /f/ y /x/ y su neutralización en $/ \phi /-$ los y las hablantes de Iquitos, al pronunciar las mismas palabras en diferentes contextos demuestran una mayor consciencia de las características del CAP (y de su estigmatización social), suprimiéndolas y sustituyéndolas con las variantes consideradas normativas.

Además, en el mismo estudio, Vallejos menciona algunos datos extraídos de un blog (que ya no se encuentra en línea) llamado Mashita Jergón, en el que tanto el autor como los lectores y los comentaristas afirmaban con orgullo su 'loretanidad', incluso en discursos metalingüísticos. En sus escritos, la autora, encuentra un uso excesivo de algunos marcadores considerados peculiares del CAP, en particular la alteración sintáctica que lleva al uso del doble posesivo, como en el ejemplo: 'su casa de Miguel'.

The hypothesis at play is that the blog might reflect an overuse of PAS features to mark an alignment towards Amazonian identity. In other words, this usage would reflect what the blog author and the readers posting comments think is characteristic of PAS. It has been suggested that the construction of self requires both explicit and implicit action and thought. It involves stressing the similarities or shared attributes around which group members coalesce. In this view, identity is the product of personal choice. Individuals actively decide to adopt or stress certain attributes. These attributes are, in this case, linguistic traits in use. (2014. p. 438)

Para la creación y el mantenimiento de la identidad amazónica, los que participan en las discusiones en el blog enfatizan, de manera más o menos consciente, algunas de las construcciones que perciben como peculiares del castellano amazónico peruano. 


\section{En la selva dicen}

A partir de las consideraciones recién mencionadas, se procedió al análisis del caso de la página de Facebook titulada "En la selva dicen" (ELSD), que empezó sus publicaciones en enero de 2016 con este mensaje " ¡Hola cumpitas! Les presento a la comunidad más bacán de la selva. Recopilamos frases, anécdotas y palabras típicas, con el objetivo de sentirnos identificados con ellas y reforzar el orgullo que tenemos por nuestro dialecto. ¡Apura cho! Manda ya tu frase por inbox pa ve en el jueis".

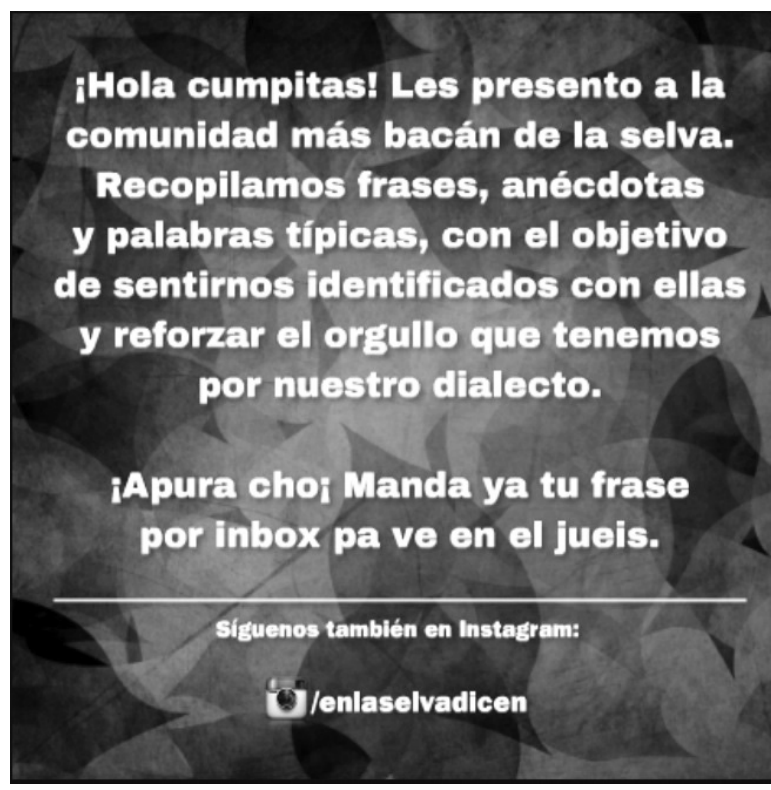

A partir de esta primera publicación los administradores emplean algunos elementos comunes en el habla amazónica: el uso de algunos vocablos reconocidos por la RAE como americanismos (cumpita, diminutivo procedente de cumpa, 
alteración de compañero; o el adjetivo bacán); la presencia de la muletilla cho, empleado como suerte de vocativo, y, por último, el intento de reproducir la locución con la eliminación de parte de los lexemas (pa en lugar de para, fenómeno, que como bien es sabido, no es exclusivo del castellano amazónico), la eliminación del último fonema consonántico en palabras agudas (ve por ver), o la adaptación ortográfica de la pronunciación asimilada de la fricativa labiodental /f/ con la velar /x/ y la diptongación epentética de /e/ $\rightarrow$ /ue/ en jueis, palabra procedente de feis, por su parte transcripción adaptada del anglicisimo face (diminutivo de Facebook). No deja de ser importante la presencia de otro anglicismo (inbox).

En efecto, puesto que el propósito explícito de los redactores es el de recopilar frases y enseñar la manera en la que los amazónicos y las amazónicas hablan, las numerosas entradas de la página ${ }^{13}$ presentan múltiples características del castellano amazónico peruano en las distintas vertientes lingüísticas.

A pesar de ser frases escritas, uno de los elementos más llamativos es la imitación de la forma de hablar, tanto a nivel fonológico como de los principales rasgos de la entonación. De esta forma, en muchas entradas la ortografía es casi fonémica, como ya se había experimentado, por ejemplo, en la novela Paiche de César Calvo de Araujo (Pau, 2017; 2018).

Entre los elementos más frecuentes destacan la asimilación del fonema $/ \mathrm{s} /$ en $/ \mathrm{J} /$, como en la palabra ashishito, modificación del adverbio así $i^{14}$ y la mencionada neutralización de

13 Hasta el momento (actualización del 25 de julio de 2018) se han publicado 545 frases.

14 Entradas (entre otras) del 18/04/16 y del 28/04/16. 
la fricativa velar / $\mathrm{x} / \mathrm{y}$ de la labiodental / $\mathrm{f} /$ en el archifonema fricativo bilabial sordo $/ \phi /$, transcrito ortográficamente con la letra jota, y la sucesiva creación de un diptongo en lugar de la vocal simple. Ejemplos de este fenómeno, además del citado jueis, son -entre otros- juebrero por febrero (13/02/16), jueliz por feliz (14/02/16; 08/03/16); juastidies por fastidies (09/03/16) y juastidio por fastidio (22/03/16); projue por profe $(06 / 07 / 16)$ o cajué por café $(26 / 08 / 16)$.

Asimismo, los administradores de la página publicaron a menudo (sobre todo en los primeros meses de ELSD) frases que tratan de reproducir la entonación peculiar del CAP y utilizaron como recurso ortográfico la repetición de las vocales en el interior de las palabras, por ejemplo: jAcaso es chaaancho esi!' (23/01/16), ...era un reeco supi que duuuro ya me estaba aguantando (08/03/16) o ; Vaaamo ya a pará esa humisha cho! $(06 / 02 / 16)$, donde, además, desaparece el fonema final tanto del verbo conjugado como del infinitivo. Tal eliminación de fonemas es frecuente en el CAP no solo al final de la palabra -como en los ejemplos anteriores o en verdá (09/02/16)-, sino también en el interior de ellas, especialmente en posición interconsonántica átona, como en vams (07/02/16).

A nivel morfológico, como acontece en todas las variantes del español peruano, uno de los elementos más recurrentes es la formación de diminutivos y las entradas de ELSD presentan un listado variado, a partir de formas más canónicas como pancito (29/08/17) o agüita (22/03/16), hasta llegar a diminutivos de diminutivos como todititos (22/03/16) o a los diminutivos de adverbios como el mencionado ashishito (18/04/16), o aquisito (17/03/18). Cabe subrayar, además, la frecuente formación de diminutivos procedentes de la unión de lexemas no castellanos (generalmente del quechua o de otra lengua in- 
dígena) con los sufijos diminutivo -ito/a e illo/a, por ejemplo, Ilullitos (01/05/17), es decir 'niñitos', ñañita (28/03/17), 'hermanita', o huambrillo (11/03/16), 'muchachito'.

La unión de morfemas quechuas y castellanos no es exclusiva de los diminutivos; otro uso muy común es el del sufijo -(t)ero con un sentido de repetición de algo, muchas veces en tono de burla. Así, encontramos en varias entradas las palabras ishpatero, 'meón' (11/03/16), del quechua ishpa, 'orina'; quichatero, 'cagón' (23/03/16), del quechua kicha, 'diarrea'; o supitera (15/09/17), también de quechua supi, es decir 'pedo'. Existen, aunque en número inferior, ejemplos de morfemas léxicos espańoles que se juntan con sufijos quechuas; entre ellos resalta la palabra buchisapa (24/05/17), formada del castellano buche (=barriga) unido al sufijo aumentativo quechua -sapa y usada para indicar una persona barriguda.

De forma similar, se da la formación de verbos denominales teminados en -ear, tanto a partir de bases quechua como de castellanas. Algunos ejemplos que aparecen entre las frases de la página son las formas ishanguearon (02/09/17), que procede del vocablo quechua ishanka, 'ortiga', y que "se refiere a la acción de golpear a alguien con ramas de ishanga [...] como castigo" (Chirif, 2016, p. 157); o también huicapear (07/04/18), procedente del sustantivo huicapa y del verbo quechua wikapay,

272 es decir arrojar, lanzar (normalmente un trozo de madera). Un ejemplo de derivación de un sustantivo castellano es el de carnavalear ((07/02/16), con el sentido de celebrar los carnavales.

Además, es muy frecuente en las entradas de la página la presencia del sufijo aumentativo -azo/a, a veces presente en la forma -aso/a; véanse por ejemplo los casos asnaso (13/01/16), asnazos (22/03/16) o haraganazo (28/03/16). 
Otros elementos morfosintácticos típicos del CAP registrados por los redactores son la presencia de pronombres reflexivos clíticos -como voyme (09/02/16)-; la alteración del orden estándar de los constituyentes de las oraciones, con la posposición del verbo como último elemento: ¿Tanto ya pues trabajan? (30/08/16), ni lavar tu calzón mapacho sabes $(02 / 11 / 17)$; o la ausencia del nexo preposicional en las perífrasis 'ir a + infinitivo': vas querer (22/03/16), vas comer $(24 / 03 / 16)$.

Por último, cabe señalar también la constante reiteración del interrogativo $i d i$ ? y de la fórmula ya vuelta, reconocida como una de las marcas características de los discursos de los amazónicos y las amazónicas: ¿ya vuelta vams ir a carnevalear? (07/02/16); ¿ya vuelta cumplimos un mes? (12/02/16); ¿ya vuelta va a ser 14 de juebrero? (13/02/16).

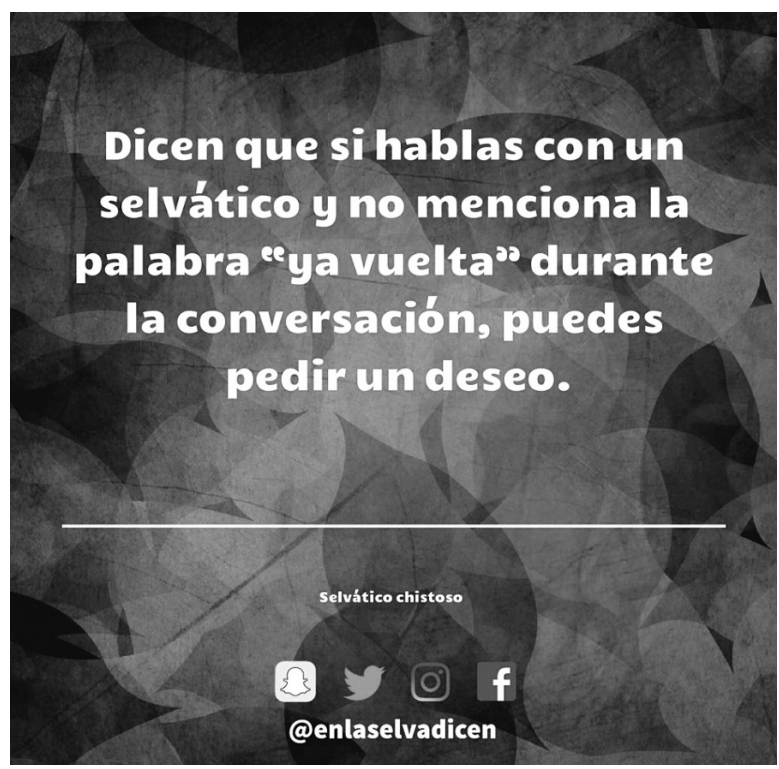


Empero, llama la atención el hecho de que en ninguna de las entradas de la página aparezca la estructura del doble posesivo, por ejemplo, 'su casa de Juan' -llamado también posesivo redundante (Rodríguez Garrido, 1982) o posesivo enfático (Escobar, 1978, p. 108) -ni la inversión del genitivo, por ejemplo, 'de Juan su casa' (Vallejos, 2004, p. 443), elementos percibidos en el imaginario común como estereotípicos del habla amazónica.

Hay que subrayar que las señales más fácilmente reconocibles del castellano amazónico peruano presentes en las entradas de "En la selva dicen" son, obviamente, las lexicales. La inmensa mayoría de las frases recopiladas proporcionan ejemplos del rico vocabulario selvático, caracterizado por la influencia del quechua y de otras lenguas indígenas (principalmente las de la familia tupí), los arcaísmos españoles, la resemantización de algunos términos del castellano estándar y la creación de palabras onomatopéyicas.

Uno de los campos semánticos más explotados es el de la gastronomía, con un rico listado de alimentos y platos típicos ${ }^{15}$, a menudo mencionados con propósitos metafóricos e irónicos, como en el caso de un poema de desamor centrado en el juane, la comida amazónica más popular, hecha de arroz envuelto en hojas: "Sin que seas juane quiero llegar a la presa

274 de tu corazón, mezclado con el arroz de tu rechazo, envuelto con las hojas de tu indiferencia" (24/06/17); o también otro poema en el que se nombran varios tipos de pescado: "Doñita linda, me da un cebiche de doncella, para mi esposa

15 Aparecen entre otros: curichi; pango de palometa; masato; juane; aguaje y aguajina; shibé; chapo; inguiri; inchicapi; siquisapa; tacacho con cecina; sachaculantro. 
bella; para mí que soy un atleta, sudado de palometa y para mi suegra, una llambina con harta espina" (15/11/17).

Sin embargo, el ámbito de mayor éxito entre todas las publicaciones es el de los tabúes: de hecho, un gran porcentaje de las frases presentes en la página tiene que ver con la corporalidad, el sexo, las necesidades fisiológicas y la escatología $^{16}$. Aun reconociendo la gracia de varias de estas entradas, cabe señalar que en muchos casos estas rozan el sexismo y la homofobia, a veces con alusiones o juegos de palabras netamente soeces.

\section{Algunas consideraciones sociolingüísticas}

Identificar y analizar la presencia de elementos característicos del castellano amazónico peruano en una página como "En la selva dicen" permite atestiguar la heterogeneidad del español en sus diversas variantes y ver cómo el empleo de las redes sociales influye sobre ellas, tanto con mecanismos conservadores como, al contrario, con tendencias homologantes. Cabe preguntarse cuál es la razón de ser de una página de este tipo y cuáles son sus objetivos a partir de un enfoque que indique cuál es la 'ideología lingüística' (Woolard, 1998, p. 3) subyacente, es decir, cuáles son esas representaciones, tanto explícitas como implícitas, que analizan la intersección entre lenguaje y seres humanos en el mundo, tomando además en cuenta que el lenguaje, a la vez que es moldeado socialmente, contribuye a dar forma a la sociedad (Fairclough, 1995,

16 Entre otros: isma, 'heces'; ocote, 'ano'; picho, 'vagina'; chucho, 'senos'; mapero, 'homosexual', pero en forma despectiva; quicha, 'diarrea'; ullo, 'pene'; supi, 'pedo'; talega, 'escroto'. 
p. 55). Parece útil abordar este asunto a través de algunos mensajes publicados por los seguidores de la página, escogidos entre los más pertinentes para este estudio y copiados directamente; solo se han eliminado las referencias explícitas a sus autores.

De hecho, la naturaleza de las redes sociales, que permiten la rápida interacción de las personas, consiente a los usuarios expresar de inmediato su opinión acerca de las entradas recopiladas. La presencia de estos comentarios -expresados en esa peculiar mezcla de oralidad y escritura que caracteriza la comunicación informal a través de internet- proporciona un interesante corpus de análisis acerca de las percepciones y de los sentimientos relativos al castellano amazónico peruano y de sus muestras presentes en ELSD.

(1) [Jajajajaja que risa me causa al leer esto ........ al principio no entendía los significados de las palabras ...... pero están lindas ..... me encantan por que, al leer con entonación causan risa ...... SALUDOS desde \#ventanilla LIMA_PERÚ]

(2) [Jaja ..me hacen reir ..extraño esas palabras ..de mi hermosa tierra]

(3) [Qué buen verso jajaja. Esas palabras ya casi las había olvidado, gracias por recordármelos jajaja]

(4) [es que asi hablamos, somos sinceros y originales.]

(5) [Hay caracho ya vuelta me hicieron acordar mis tiempos cuando era medio shicshi y como extraño mi selva hermosa. Estoy muy orgullosa d ser d la selva y por hablar asi no me considero ignorante. Saludos a todos q viven y viviran orgullosos d ella] 
(6) [Soy selvática, soy maestra, yo les enseño a mis alumnos a expresar las palabras correctamente, pero jamás nunca les llamaria ignorantes,también les digo que ser selvático es ser único, nuestra forma de hablar es única,tiene esa frescura que a más de uno le saca una carcajada, así somos es nuestra esencia,y hay que aceptanos, conozco hermanos de la costa y sierra que también pronuncian mal muchas palabras pero no les tildo de ignorantes por sé que el medio geográfico condiciona hasta la forma de hablar de las personas,sé de reglas ortográficas pero también sé que mi selva es única.]

Los comentarios (1) y (2) aun refiriéndose a la ironía que caracteriza la mayoría de las publicaciones, nos remiten a las observaciones de Jara Yupanqui (2012, p. 459), según la cual algunos jóvenes percibirían el léxico típico del CAP como ‘gracioso' y que tal impresión se debería a una reducción en la frecuencia de uso de esas palabras respecto a las generaciones anteriores. La afirmación de Jara es corroborada tanto por el comentario (2), en el que se afirma: "extraño esas palabras", como por el (3): "Esas palabras ya casi las había olvidado, gracias por recordármelos”.

Sin embargo, volviendo a 'lo gracioso' de las frases, los dos comentarios mencionados difieren 'por qué' lo son: en (2), comentario dejado claramente por una persona procedente de la selva, la risa está conectada justamente al sentimiento de añoranza; mientras que en (1), comentario procedente de un distrito de Lima, la risa es causada por la lectura "con entonación", es decir, es la típica inflexión amazónica lo que es percibido como gracioso. 
Como señala Patrucco (2002, citado por Fiorentino, 2005), uno de los tipos canónicos de páginas web que se ocupan de variantes dialectales es el de las "irónicas”, es decir páginas en las que el dialecto es usado a menudo como vehículo de chistes o juegos de palabras. ELSD se ajustaría a esta clasificación $y$, puesto que el conocimiento compartido de una lengua o de algunas características de la lengua puede contribuir a crear efectos humorísticos (Ritchie, 2010, p. 33), esto justificaría también el uso frecuente del léxico referido a los temas tabúes, muchas veces incomprensible para hispanohablantes no amazónicos.

Los comentarios metalingüísticos esbozan un sentimiento de identificación y de orgullo, el de pertenecer a un 'nosotros' amazónico en contraste con los ‘otros' (Hall, 1996), principalmente limeños. Tanto el mencionado mensaje inaugural de la página, como los comentarios (4), (5) y (6) lo atestiguan: "Estoy muy orgullosa d ser d la selva y por hablar asi no me considero ignorante". Esta afirmación concluye una larga discusión empezada por una usuaria, también procedente de la selva, que usaba ese adjetivo para calificar a los autores de las entradas y a los seguidores de la página. En la discusión intervinieron los mismos administradores, quienes comentaron de esta forma:

(7) [No los llamaría "ignorantes", [...] porque ignorancia es no saber reconocer tu cultura y tus raíces, dejarlos atrás o avergonzare de ello. En la selva dicen muestra parte del dialecto que se utiliza, con el objetivo de promover el orgullo que tenemos por él y entretenernos]

Planteo que este intento de construir orgullosamente la identidad amazónica a través del lenguaje es la razón por la que en 
el corpus de frases recopilado y publicado no aparece ninguna referencia al doble posesivo o a la inversión del genitivo. Esas construcciones sintácticas, en efecto, ya han dejado de ser percibidas como meros 'indicadores' del habla amazónica, para convertirse en estereotipos (Labov, 1972) estigmatizados, empleados frecuentemente como burlas. Esta también podría ser la razón que ha llevado (a partir de finales de 2016) a la desaparición de entradas en las que se emplea el recurso de la repetición de las vocales para imitar la entonación.

Con la eliminación de las marcas más fácilmente reconocibles y connotadas negativamente del castellano amazónico peruano ${ }^{17}$, los administradores tratan de dar forma (más o menos conscientemente) a un nuevo imaginario, más neutro, sobre los y las habitantes de la selva.

\section{Conclusiones}

El análisis de la página Facebook "En la selva dicen" ha permitido la identificación, en la mayoría de las entradas, de algunas de las principales características del castellano amazónico peruano. A nivel fonológico, los autores buscan reproducir ortográficamente algunos de los rasgos peculiares de la pronunciación y de la entonación de los y las hablantes, mientras que a nivel morfosintáctico, atestiguan fielmente las recíprocas influencias entre el castellano y las lenguas autóctonas con la presentación, por ejemplo, de varias palabras derivadas de elementos de distinta procedencia o de oraciones en las que el orden de los constituyentes se aleja del que se

17 En esta misma dirección iría la entrada publicada el 3 de noviembre de 2017, que dice "Se nos podrá salir un supi, pero jamás un 'haiga"”. 
considera estándar. El ámbito que ha resultado ser el más llamativo es el léxico. De hecho, son numerosas las referencias a temas de la vida cotidiana como la gastronomía y, sobre todo, a tabúes como la corporalidad y el sexo, empleados con fines humorísticos a pesar de las frecuentes alusiones de mal gusto.

Al profundizar el análisis sociolingüístico, han resultado casi ausentes algunos elementos que en el imaginario peruano son estereotípicos del habla amazónica. En el corpus no se presenta ningún ejemplo de doble posesivo o de inversión del genitivo, mientras que los recursos utilizados para reproducir la típica entonación desaparecen después de los primeros meses de vida de la página.

Esto se debe, en nuestra opinión, al propósito fundamental explicitado en varias ocasiones por los creadores de la página: (auto)representarse y (auto)identificarse con orgullo como peruanos amazónicos y peruanas amazónicas, desprendiéndose de ideas preconcebidas y de prejuicios.

Recibido: 26 de agosto del 2018.

Aprobado: 19 de diciembre del 2018.

\section{Bibliografía}

Arroyo, J. M.

(1977). Nota sobre el habla de Loreto. Conocimientos. Revista de la Universidad Nacional de la Amazonia, 1(3), 7-12.

Barraza, Y.

(1998). Apuntes sobre gramática del castellano de Iquitos (Tesis para optar el grado académico de magister en lin- 
güística). Universidad Nacional Mayor de San Marcos, Lima.

Berruto, G.

(2004) Prima lezione di sociolinguistica. Bari: Laterza.

Chirif, A.

(2016). Diccionario amazónico. Voces del castellano en la selva peruana. Lima: Lluvia editores/CAAAP.

Escobar, A.

Variación sociolingüistica del castellano en el Perú. Lima: IEP.

Escobar, A. M.

(2010). Variación lingüística en español. En J. I. Hualde, A. Olarrea, A. M. Escobar y C. E. Travis, Introducción a la lingüistica hispánica. New York: Cambridge University Press.

Fairclough, N.

(1995) Media Discourse. London: Arnold.

Falcón Ccenta, P. M.

(2012). Características del léxico verbal en el castellano de los indígenas bilingües amazónicos. Lengua y Sociedad. Instituto de Lingüistica Aplicada, 12(1), 141-152.

Falcón Ccenta, P. M., Chumbile, R. y Canturín, J.

(2012). Características morfosintácticas del castellano amazónico en hablantes indígenas bilingües en Lima. Escritura y Pensamiento, 15(30), 79-112.

Fiorentino, G.

(2005). Dialetti in Rete. Rivista Italiana di Dialettologia, 29, 111-149. 
García, J.

(2015) Nota de los editores. En L. Castonguay, Vocabulario regional del oriente peruano. Iquitos: CETA.

García, M.

(2016) The intonational patterns of the Peruvian Amazonian Spanish. Rising Accents and Segmental Factors (Tesis para optar el grado de doctor). The Ohio State University.

Hall, S.

(1996) Introduction: who needs identity? En S. Hall y P. Du Gay (Comps.), Questions of cultural identity (pp. 1-17). London: Sage Publications.

Hudson, R.

Sociolinguistica. Bologna: Il Mulino.

Jara Yupanqui, M.

(2012) Peruvian Amazonian Spanish: linguistic variation, language ideologies and identities. Sociolinguistic Studies SOLS, 6(3), 445-469.

Jara Yupanqui, M. y Valenzuela, P.

(2013) El uso del perfecto en secuencias narrativas en el español peruano amazónico: el caso de Jéberos. Lexis, $37(1), 33-70$.

282 Labov, W.

(1972) Socioliguistic Patterns. Philadelphia: University of Pennsylvania Press.

Marticorena, M.

(2010) El castellano amazónico del Perú. Iquitos: Instituto de Investigaciones Educativas e Históricas de la Amazonía Peruana. 
Matthews, P. H.

(1979)

Generative Grammar and Linguistic Competence. London: Allen \& Unwin.

Patrucco, E.

(2002) Dialetto on line. Italiano \& oltre, 17, 140-144.

Pau, S.

(2017) Modelos para la superación de la crisis del sistema capitalista: Paiche y E venne il sabato, novelas amazónicas. Revista de Critica Literaria Latinoamericana, 62 (86), 277-300.

Pau, S.

(2018) El castellano amazónico en la novela "Paiche". Análisis lingüístico. Inédito.

Pérez Falcón, T.

(2015) El adverbio en el castellano de Iquitos (Tesis para optar el título de licenciatura. Universidad Nacional Mayor de San Marcos, Lima.

Ramírez, L. H.

(2003) El español amazónico hablado en el Perú. Hacia una sistematización de este dialecto. Lima: Juan Gutemberg Editores

Ritchie, G.

(2010) Linguistic factors in humor. En D. Chiaro (Comp.), Translation, Humor and Literature (pp. 33-48). London: Continuum.

Rodríguez Garrido, J. A.

(1982) Sobre el uso del posesivo redundante en el español del Perú. Lexis, 6(1), 117-123. 
San Román, J.

(1975). Perfiles históricos de la Amazonía peruana. Iquitos: CETA.

Santos Granero, F. y Barclay. F.

(2002). La frontera domesticada. Historia económica y social de Loreto 1850-2000. Lima: Fondo Editorial PUCP.

Solís Fonseca, G.

(2009) Perú amazónico. En Atlas sociolingüistico de pueblos indígenas en América Latina. Cochabamba: UNICEF y FUNPROEIB Andes.

Vallejos, R.

(2014) Peruvian Amazonian Spanish. Uncovering variation and deconstructing stereotypes. Spanish in Context, 11(3), 425-453.

Vallejos, R.

(2017) Nominal possession in Peruvian Amazonian Spanish: the role of animacy in the emergence of grammar. Inédito Recuperado de: https://wwwacademia. edu/31475095/Nominal_possession_in_Peruvian_Amazonian_Spanish_the_role_of_animacy_in_the_emergence_of_ grammar

Varese, S.

284

(1973) La sal de los cerros. Lima: Retablo de Papel.

Woolard, K.

Introduction: Language Ideology as a Field of Inquiry”. En B. Schieffelin, K. Woolard y P. Kroskrity (Comps.). Language Ideologies. Practice and Theory. New York: Oxford University Press. 